ARTíCULOS DE INVESTIGACIÓN

\title{
Pedagogía de la pregunta en la enseñanza-aprendizaje del derecho como innovación metodológica para desarrollar el pensamiento crítico-complejo: Un análisis de caso
}

\author{
Pedagogia da pergunta no ensino-aprendizagem do direito como inovação metodológica \\ para o desenvolvimento do pensamento crítico-complexo: Um estudo de caso
}

\section{Pedagogy of the question in law teaching-learning as methodological innovation to develop critical-complex thinking: case study}

\author{
Taeli Gómez Francisco \\ Universidad de Atacama, Chile \\ Juan Rubio González \\ Universidad de Atacama, Chile \\ Walter González Morales (iD \\ Universidad de Atacama, Chile
}

\begin{abstract}
RESUMEN Este trabajo tiene como objetivo contribuir a la docencia jurídica. Para lograrlo, se dan a conocer los alcances de una experiencia de intervención realizada en la carrera de Derecho de la Universidad de Atacama en Chile, donde se desarrolla la pedagogía jurídica basada en la pregunta como una innovación metodológica para el desarrollo de pensamiento crítico-complejo en el estudiante de derecho. A partir de esta estrategia pedagógica que buscaba intencionar la pregunta como táctica problematizadora, se analizaron los principales fundamentos de la pedagogía de la pregunta en la enseñanza-aprendizaje jurídico, y utilizando técnicas e instrumentos de las metodologías participativas se sistematizaron antecedentes relevantes emanados de la experiencia y de la percepción de los estudiantes, los que proporcionaron insumos para complementar el análisis de este trabajo. Se discute y concluye que se requiere prestar atención a los fundamentos epistémicos, políticos y pedagógico-afectivos como factores indispensables para que una pedagogía de la pregunta se concretice y se transforme en la base del desarrollo de pensamiento crítico-complejo en el estudiante de derecho.
\end{abstract}


PALABRAS CLAVE Pedagogía de la pregunta, pensamiento crítico-complejo, enseñanza del derecho.

RESUMO Este artigo tem como objetivo contribuir para o ensino jurídico, para o qual, o escopo de uma experiência de intervenção realizada na Faculdade de Direito da Universidade de Atacama, Chile, onde a Pedagogia Jurídica Baseada no Pergunta, como inovação metodológica para o desenvolvimento do pensamento crítico-complexo no estudante de Direito. A partir dessa estratégia pedagógica realizada, que, de maneira particular, buscou considerar a questão como uma tática problemática, são analisados os principais fundamentos da Pedagogia da Questão no ensino-aprendizagem legal, e sistematizados, utilizando técnicas e instrumentos de metodologias participativas. antecedentes relevantes emanados da experiência e da percepção dos alunos que dela participaram, aqueles que fornecem subsídios para complementar a análise deste trabalho. Argumenta-se e conclui que é necessário prestar atenção aos fundamentos epistêmicos, políticos e pedagógicos-afetivos, como fatores indispensáveis para que uma Pedagogia da Questão seja concretizada e transformada na base do desenvolvimento do pensamento crítico-complexo no estudante de Direito .

PALAVRAS-CHAVE Pedagogia da pergunta, pensamento crítico-complexo, ensino de direito.

ABSTRACT The objective of this research is to contribute in law teaching, conveying the intervention experience in the Law Career in Universidad de Atacama, Chile. Here, the Law Pedagogy Based in Question was developed as a methodological innovation to develop the critical and complex thinking in law student. From this pedagogical strategy, that in a particular way sought to intend the question as a problematizing tactic, the foundations of Question Pedagogy in teaching-learning law are analyzed, using techniques and instruments of participative methodology, relevant information emanated from the experience and the perception of the students who participated was systematized. This give inputs to complete the analysis. In this research there is a discussion and conclusion that it is necessary to give attention to epistemic, political and pedagogical-emotional foundations as essencial factors to put in practice a Question Pedagogy as the base of the development of critical-complex thinking in Law students.

KEYWORDS Question pedagogy, vritical-vomplex thinking, law teaching.

\section{Introducción}

En la enseñanza del derecho tradicionalmente se ha incentivado una práctica educativa que promueve la pasividad receptiva del conocimiento de las ciencias jurídicas, avalando lo que algunos autores denominan como una pedagogía de la respuesta (Freire y Faundez, 2013; Tapia, 2014). Lo anterior se vislumbra en las prácticas educativas desarrolladas desde paradigmas clásicos y metodologías mecanicistas y lineales, 
que disponen tanto la organización de las clases como los fundamentos epistémicos y políticos que están involucrados en los contenidos dictados, en la lógica de promover una noción de objeto de conocimiento dado (Gómez, 2018), y una verdad externa y distinta del sujeto que conoce.

Se ha desarrollado la práctica de transmitir los contenidos previamente ordenados y clasificados como texto sin contexto, muchas veces entregando respuestas que los estudiantes - y a veces docentes - no se han preguntado (Freire y Faundez, 2013), con lo cual se reproduce la lógica de una pedagogía de la respuesta y un método de enseñanza claramente exegético (Gómez, 2014). Al, el Proyecto Tuning para América Latina (2004-2007), después de indagar con un grupo de trabajo conformado por dieciocho universidades sobre la formación de los futuros abogados, reafirma lo anterior y señala que se mantiene un énfasis exacerbado a la retención de información jurídica (Beneitone y otros, 2007).

Estos mismos diagnósticos han tenido lugar desde hace algunos años en la comunidad jurídica nacional e internacional que imparte docencia universitaria, ${ }^{1}$ (Mareto y Bussinguer, 2016; Santos y Macedo, 2018; Illera, 2017; Osorio, 2013; Fach, 2012; Mastache y Devetac, 2017; Rivera, 2014) admitiendo principalmente que desarrollar la creatividad para elaborar un caso, así como la habilidad para argumentar y diseñar estrategias, resulta ser un asunto consustancial a la praxis de un abogado. Estos factores, en última instancia, están relacionados y tienen un punto de inicio desde la acertada problematización de la práctica jurídica, que se operativiza a partir de la formulación de preguntas precisas con a los hechos y a las normas jurídicas en cuestión.

Desde esa perspectiva, se vislumbra una estrecha relación entre la capacidad de problematizar y el desarrollo de pensamiento crítico-complejo en los sujetos toda vez que la acción problematizadora es un proceso que desencadena la generación de conocimiento científico y para lo que, de acuerdo con Sánchez-Puentes (1993), se debe producir la necesaria desestabilización y cuestionamiento de la labor y el rol que están desarrollando los sujetos. Desde esta flexibilidad, resulta posible la apertura cognitiva que permite clarificar y ordenar las ideas, identificar y diferenciar los elementos relevantes de los accesorios y contextualizar las temáticas estudiadas como un acto propio y no dado por otros, como objeto de conocimiento. Todas estas características enunciadas, propias del proceso problematizador, corresponden a la génesis del pensamiento crítico-complejo, de ahí que siguiendo a Savater (1999) en la enseñanza jurídica parece preferible mantener abiertas grandes preguntas antes que concentrarse en pequeñas respuestas.

1. Abogados-docentes universitarios, así como profesionales de otras disciplinas como la pedagogía, sociología y psicología reunidos en Congresos de Pedagogía y Enseñanza del Derecho realizados en la Universidad de Chile desde el 2010-2019; Universidad Autónoma de México (2014); Universidad de Buenos Aires (2016); Brasil (2018); entre otros espacios de diálogo han generado este diagnóstico. 
Asumiendo este panorama, en la carrera de Derecho de la Universidad de Atacama en Chile, en el contexto de un Programa para la Enseñanza Integral del Derecho, ${ }^{2}$ se implementa una estrategia pedagógica denominada pedagogía jurídica basada en la pregunta, desarrollada fundamentalmente en la asignatura de Derecho Civil I y II. Esta se planteó como primer objetivo explorar la existencia de un aula problematizadora y, en caso de no haberla, indagar sobre cuáles eran las razones.

La estrategia pedagógica implementada desde el Programa para la Enseñanza Integral del Derecho es contextualizada en la declaración de un perfil de egreso del licenciado en ciencias jurídicas de la Universidad de Atacama que admite formar habilidades para argumentar y razonar jurídicamente, por lo que se intenta rescatar la importancia de la pregunta en la enseñanza del derecho y la relevancia que tiene para la formación de un futuro abogado crítico-complejo. Ante esto es necesario, además, establecer lineamientos estratégicos que propongan una mirada inter, multi y transdisciplinaria, que permita plasmar en la práctica un sujeto crítico y reflexivo de la realidad social y, por cierto, en lo relacionado a la interpretación y aplicación de la normativa jurídica.

Para desarrollar esta estrategia pedagógica la experiencia se fundamenta en una metodología participativa, en específico centrada en el modelo de la investigaciónacción-participación (Elliot, 1994; Villasante, Montañés y Martí, 2000; Latorre, 2003), el cual permitió desplegar diversas técnicas e instrumentos de carácter cuantitativo, cualitativo y participativo, destacándose la observación participativa y el diario de campo sumado a las actividades propias de la asignatura. Estos elementos permitieron sistematizar y evaluar la experiencia desarrollada durante tres años académicos $y$, sobre todo, conocer la percepción de los estudiantes de derecho con a la pertinencia, pedagogía y capacidades para realizar interrogantes durante las clases, entendiéndolo como una proyección de su futura práctica profesional. Parte de estas percepciones estudiantiles se dan a conocer en este trabajo, ya que resulta interesante poder evaluar las posturas epistemológicas que sustentan la pedagogía de la pregunta a la luz del sentir de los protagonistas del proceso de enseñanza-aprendizaje.

En ese sentido, los antecedentes, la información, los datos recolectados durante este período y la experiencia desarrollada fueron conectados e interpretados a partir de los diversos postulados teóricos y epistemológicos, que han venido nutriendo la

2. El Programa para la Enseñanza Integral del Derecho surge en 2013 no solo por la preocupación de mejorar el proceso de enseñanza y aprendizaje, sino en un contexto sintético de cambios en la enseñanza del derecho en general, de nuevos escenarios epistemológicos y ejecución de paradigmas complejos, de políticas educativas y aportes en las ciencias de la educación, y valoración de aprendizajes socioconstructivistas. Se propusieron las líneas estratégicas de enseñanza, investigación estudiantil e investigación situada. Para organizar una implementación operativa se diseñó la ejecución por trazas, una de las cuales se denomina pedagogía jurídica basada en la pregunta. 
enseñanza jurídica y que dan cuenta de las bases epistemológicas desde donde surgen las teorías relacionadas a la pedagogía basada en la pregunta. Es así como en el desarrollo e implementación de esta estrategia pedagógica se obtienen algunos hallazgos preliminares, los cuales permiten entregar ciertos indicios sobre los factores que estarían impidiendo desarrollar aulas jurídicas problematizadoras, pero también constatando la conveniencia de fortalecer una intencionalidad pedagógica de la pregunta y, por sobre todo, admitir tres fundamentos complementarios que deben estar presentes en los procesos de enseñanza-aprendizaje que se propongan cualidades problematizadoras: el fundamento epistemológico, el político y una pedagogía con atención al ámbito afectivo del estudiante.

\section{Aproximación a la pedagogía basada en la pregunta}

Durante el proceso de problematización, en el estudiante de derecho se produce la confluencia de tres ámbitos relevantes a considerar: la acción práctica, la cognición y el desarrollo afectivo. En efecto, este proceso pone en marcha un conjunto de factores que le permiten producir una meta mirada, donde el estudiante en una primera fase de acercamiento ha de conocer y aceptar sus deficiencias, sus restricciones personales y teóricas sobre los objetos estudiados. Al, García y García (2005) plantean que, debido a la inexperiencia del aprendiz, en un primer momento de la problematización tiende a buscar y encontrar salidas cómodas al generarse preguntas, vale decir, realiza cuestionamientos simples, poco profundos, lo cual obviamente tiene pocas posibilidades de lograr respuestas novedosas y fecundas. A medida que establece una práctica y le da sentido estratégico a la pregunta, el estudiante profundizará intelectualmente y permitirá esencializar las preguntas (García y García, 2005).

Lo cierto es que no tiene nada nuevo el plantearse la importancia de la pregunta como metodología para la formación de estudiantes, en especial en la docencia universitaria. Diversas son las metodologías que han contribuido a desarrollarla. Ejemplo de lo anterior es la propuesta de aula socrática, reconocida como una práctica pedagógica destinada a promover el pensamiento crítico y a la cual Martínez (2006) define como aquel método que desde la ironía se complementa con la mayéutica o arte de comadrona de los espíritus, desarrollándose como un procedimiento por el cual Sócrates - con sus hábiles interrogaciones, refutación e investigaciones- provoca a los interlocutores para hacerles encontrar lo que ellos mismos tienen en el espíritu, dándolos a la luz de su propio pensamiento.

Diversos autores reconocen que esta estrategia, por un lado, genera pensamiento crítico (Betancourth, Insuasti y Riascos, 2012; López, 2012), pero además logra fortalecer un pensamiento reflexivo, argumentativo y propositivo en los estudiantes (Álvarez, 2013). En esa línea, Elder y Paul (2002) señalan que el aula socrática también promueve un pensamiento indagante, analítico, sintético, creativo, que en 
última instancia forma conexiones. Estos autores también abordan la pregunta como parte de una estrategia psicopedagógica, considerando que contribuye a la formación de un pensamiento crítico. Así al menos lo establecen a partir del desarrollo del movimiento Critical Thinking, donde advierten que el hacer preguntas esenciales permite reconocer las esencias y promueve un pensamiento disciplinado, preparado para aprender (Elder y Paul, 2002).

Una perspectiva similar la desarrolló Paulo Freire, quien además inserta la pedagogía basada en la pregunta en el paradigma de la problematización. Al , el abogado y pedagogo brasileño establece categóricamente que «iel educador, de manera general, ya trae la respuesta sin que se le haya preguntado nada!» (Freire y Faundez, 2013: 69), configurando esta lógica psicopedagógica como una "castración de la curiosidad» (Freire y Faundez, 2013: 69). La propuesta de Freire en ese contexto no se orienta solo en la línea de proponer un método pedagógico y didáctico, sino que además contiene una definición epistemológica fundamental a la que alude cuando señala que la verdad se encuentra en el devenir del diálogo (Freire y Faundez, 2013).

Considerando los argumentos esgrimidos en las diversas líneas teóricas existentes para admitir la pregunta como práctica pedagógica problematizadora y generadora de pensamiento crítico-complejo, se pueden agrupar y expresar tres fundamentos complementarios, que no solo la sustentan como una estrategia educativa válida, sino que se convierten en requisitos consustanciales para lograr que una pedagogía basada en la pregunta tenga impacto en la formación del estudiante de derecho.

\section{Fundamentos epistemológicos de la pedagogía de la pregunta}

Para lograr que una pregunta emerja como parte de un contexto problematizador y no sea el resultado de una mera inspiración episódica, se requieren cambios epistemológicos esenciales.

En primer lugar se requiere de una revisión epistemológica que plantee que el sujeto de conocimiento no es independiente del objeto a conocer. Conocido en epistemología como la relación sujeto-objeto, ha puesto en tela de juicio la dualidad cartesiana, donde el sujeto pretende ser capaz de observar el mundo objetivamente, independientemente de su propia mirada (Najmanovich, 2005). Es decir, si el objeto de conocimiento - en este caso el derecho- con el que el estudiante se va a relacionar se plantea como algo objetual, no da posibilidad a participar en la construcción del objeto, perdiendo sentido su problematización. De ahí que si bien resulta un avance pedagógico abrir espacios a preguntas, se debe empoderar al estudiante como sujeto con potencialidad creadora en una relación dialéctica interdependiente sujeto-objeto.

En segundo lugar, el objeto de conocimiento ha perdido el reinado de un objeto sustancial y de la unidad elemental (Morin, 2001), dejando de ser representado como la unidad dada para pasar a ser concebido como una trama (Capra, 1998). Así, solo 
desde esta perspectiva se posibilita una relación de constructividad del objeto de conocimiento y donde la problematización del sujeto pueda impactar creativamente. En este sentido, sí se fomenta la pregunta en el aula, pero no se permite problematizar el objeto de conocimiento - el derecho- porque está puesto para ser conocido, se está promoviendo solo una ilusión metodológica.

En tercer lugar, para permitir la construcción del conocimiento en un contexto dialógico se requiere también de un cuestionamiento epistemológico de la verdad. Al respecto, Rigoberto Pupo (2004a) ha abordado esta temática desde la filosofía y ha señalado que la verdad es un proceso y resultado aprehensivo humano, por lo tanto, representa un saber profundo, construido por la actividad del hombre en relación con el mundo (Pupo, 2004a), y donde propone a la educación como una gran metáfora de la vida que debe lograr un saber integral incluyente en la búsqueda de la verdad (Pupo, 2004b).

Así, para abordar una pedagogía de la pregunta jurídica como actividad problematizadora con impacto en el proceso educativo del estudiante de derecho, se requiere de una revisión epistemológica que promueva a un sujeto activo en la construcción del conocimiento y de un objeto de estudio efectivamente problematizable. Esta posición es fundamental para desarrollar en los estudiantes una apuesta a relacionarse con los nuevos tiempos de incertidumbre (Gómez, 2015), que exigen de sujetos capaces de crear y, al mismo tiempo, les otorga herramientas para deconstruir tanto parámetros como paradigmas que limitan la búsqueda de soluciones a los nuevos y emergentes problemas.

\section{Fundamentos políticos de la pedagogía de la pregunta}

En esta consideración se comprende la pregunta como práctica pedagógica, es decir, como una manera de replantear el aula con profundización a la intersubjetividad, como modo idóneo de la formación humana (Pupo, 2004b). En esa línea, se plantea que fomentar el lenguaje puede generar acciones creativas e incluso reconocer en el otro-estudiante una fuente validada de producción de conocimiento y, además, una decisión dispuesta del estudiante, ya que al preguntar se elige qué se quiere saber y qué conocimiento es el pretendido (Vargas y Guachetá, 2012).

Desde esa perspectiva, tanto el hábito de preguntar y el desarrollo de una habilidad hacia la pregunta promueven en el estudiante una interacción activa y responsable con el contenido que debe comprender y crear. De ahí que la democratización del aula con la promoción de una pedagogía de la pregunta enriquece el proceso pedagógico, ya que brinda al profesor un ángulo distinto mientras que un docente directivo y autoritario limita también su propia curiosidad y la profundización crítica (Vargas y Guachetá, 2012). Al respecto, González (2012) plantea como aspiración de un sistema político democrático la formación de abogados críticos, para lo cual, en su proce- 
so formativo, es necesario considerar que la interacción estudiante-profesor intente materializar el cambio desde la respuesta pedagógica a la pedagogía de la pregunta.

En esa línea, la formación dinámica que el constructivismo pedagógico propone debería responder a la pregunta de cómo aprenden juntos estudiantes y profesores (González, 2012), toda vez que la tendencia en la docencia jurídica actual parte del diagnóstico de una práctica que limita al estudiante a pensar, proyectar y teorizar, ya que está centrado en memorizar. De ahí que González (2012) hable de un docente que realiza monólogos temáticos, donde no toma en cuenta otros puntos de vista, ni los de otra disciplina; no problematiza, sino que es un mero transmisor de información, con lo cual se perpetúa una tendencia reaccionaria que acentúa la cultura de la sumisión y del silencio del aprendiz (Zuleta, 2005).

\section{Fundamentos pedagógicos-afectivos de la pedagogía de la pregunta}

Estos promueven una manera de disponerse afectiva y motivadamente en el aula, en tanto constituyen una dinámica abierta que origina sujetos presentes y activos. En esto es relevante tener presente que hoy se reconoce la relación entre procesos cognitivos y afectivos como señala González-Rey (2000), quien advierte que esta idea ya está en Vigotsky en las primeras décadas del siglo XX, quien a su vez entiende las emociones humanas como «una tendencia a actuar en una dirección determinada» (2004:40), lo cual establece la existencia de una estructura afectiva que condensa las fuerzas integradoras y motivacionales de la conciencia humana, donde las emociones de un sujeto serían una actividad orientadora de su accionar. De ahí que este fundamento surja como un factor determinante para el desarrollo de cualquier estrategia de aprendizaje, sobre todo en la pedagogía de la pregunta, donde se requiere que el aprendiz tenga un papel activo, crítico y participativo.

Lo cierto es que el creciente interés por considerar que en los procesos de aprendizaje intervienen factores cognitivos y afectivos en una relación dialéctica se debe a la llamada «revolución cognitiva», originada en la década del sesenta del siglo pasado. Desde ahí se han puesto en tela de juicio los modelos de aprendizaje de «caja negra», donde se consideraba una linealidad y mecanicidad del proceso educativo sustentado solo en dos variables: de entrada (enseñanza) y salida (rendimiento), sin considerar la diversidad de factores que emergen en el sujeto que aprende (Schulman, 1986).

En las últimas décadas del siglo pasado y desde diversas corrientes, se afianza la idea sobre la necesidad de conjugar el componente cognitivo como inseparable del afectivo, sobre todo en vista de los procesos de enseñanza-aprendizaje. A modo de ejemplo, Bloom (1976) establece la existencia de tres factores determinantes en los resultados del aprendizaje: la calidad de enseñanza, las conductas cognitivas del alumno y las características afectivas del estudiante, mientras que en el último tiem- 
po diversos estudios han examinado la influencia que ejercen determinadas disposiciones afectivo-motivacionales sobre el funcionamiento cognitivo.

En ese sentido, es válido considerar el aspecto pedagógico-afectivo como fundamento de la pedagogía de la pregunta toda vez que siendo la afectividad una estructura emocional que moviliza al pensamiento y la práctica del estudiante (Rubio, 2016a) debería ser una dimensión basal, imposible de soslayar en cualquier tipo de proceso de enseñanza-aprendizaje, sobre todo en los que intencionen participación, autonomía, posturas y argumentación crítica.

\section{Antecedentes relevantes emanados de la experiencia desarrollada}

Bajo la metodología investigación-acción-participación, el Programa para la Enseñanza Integral del Derecho desarrolló la estrategia didáctica pedagogía jurídica basada en la pregunta implementada en la asignatura de Derecho Civil I y II en un período de tres años, intentando promover en el estudiante la disposición por la pregunta. A partir de esto, se buscó alcanzar los siguientes objetivos: a) contribuir a la formación de un estudiante problematizador; b) la formación de pensamiento crítico-complejo desde la base de relaciones intersubjetivas conformadas en comunidades de aprendizajes; c) intencionar un clima socializador del proceso enseñanza-aprendizaje marcado por establecer la escucha activa de los compañeros de aula; y d) el fortalecimiento de la conexión de currículo, lo que implica superar el reduccionismo de la pregunta por la duda para avanzar hacia la complejidad de la misma como revisión epistémica, fomentando un pensamiento capaz de deambular entre diálogos disciplinarios sobre la base de superar la perspectiva política de un aula con verticalidad autoritaria de escucha pasiva.

Para lograr lo anterior, se diseñó una tipología de la pregunta que permitió conocer y operacionalizar el proceso como se explica en la tabla 1.

Tabla 1. Tipologías de preguntas y sus objetivos.

Tipo de pregunta

Pregunta simple: no agrega conocimiento nuevo.

nta simple-adicionada: si bien no agrega conocimiento nuevo, aporta una asociación, ejemplifica o compara.

Pregunta creadora: utiliza la información del profesor para la creación de un nuevo conocimiento. Pregunta argumentativa: busca poner en duda, refutar, discrepar la opinión del otro.

\section{Objetivos}

Aclaración conceptual, obtener información sobre algún contenido en específico.

Ejemplo: ¿Qué significa ? ¿Puede explicar?

La aclaración conceptual la realiza utilizando, comparando o contrastando conocimiento anterior.

Ejemplo. ¿El artículo que señala tiene la misma estrategia legislativa del artículo...?

Proporcionar un aporte para ampliar el conocimiento propio y/o colectivo. Ejemplo: ¿El derecho, de acuerdo a lo que dice, es ciencia? ¿Usted qué opina? Intenta argumentar una idea, opinión, interés o ideología desde la pregunta para debilitar o desacreditar un argumento contrario. Ejemplo: El autor parte de un supuesto patriarcal, ¿podría desde otra perspectiva fundamentarse la institución jurídica que usted señala? 
Considerando la tipología de la tabla 1, se desarrolló una intervención por un período de diez semanas en la cual se diseñó una bitácora de trabajo que debía ser completada por los estudiantes. Esta se les entregó para ser llenada de manera individual y reflexivamente después de la clase o en su tiempo de trabajo autónomo, la cual fue solicitada al término de la undécima clase. La bitácora contenía el tema de la clase, las preguntas que hizo o pensó hacer el estudiante, la identificación del tipo de pregunta y el aporte que logró para sí y/o la clase.

Como resultado del ejercicio realizado, según la tabla 2, se logró establecer que de una muestra de 30 estudiantes informaron un total de 58 preguntas, 47 de ellas quedaban incorporadas dentro de la tipología de la pregunta simple, 9 en la simple adicionada, 2 en la creadora y/o en la argumentativa.

Tabla 2. Resumen de la tipología de preguntas realizadas por los estudiantes en el período

\begin{tabular}{|lllll|}
\hline Tipos de preguntas formuladas & Simples & Simple-adicionada & Creadora & Argumentativa \\
Total & 47 & 9 & 2 & 0 \\
\hline
\end{tabular}

En una segunda fase, con el objetivo de promover cambios que incorporaran la pregunta problematizadora en el aula, se implantaron una serie de estrategias pedagógicas y didácticas como el aula socrática, la cual a partir de preguntas va generando nuevas interrogantes a la vez que profundiza hacia nuevas reflexiones, sin embargo, el proceso demostró que a pesar de los avances en términos de participación e incluso de motivación, los estudiantes no lograron cambios significativos, ya que los instrumentos evaluativos y de control realizados demostraron que las tipologías de preguntas seguían focalizándose solo en simples y simples-adicionadas. Una de las razones para no preguntar, ergo, no escalar en la complejidad de las preguntas esgrimidas mayoritariamente por los estudiantes, fue su innecesaridad. Esto se observa en la figura 3 del apartado siguiente, donde se dan a conocer los resultados ante la consulta sobre la desmotivación para realizar preguntas por parte de los estudiantes.

\section{Percepciones de los estudiantes de la carrera de Derecho y de la Universidad de Atacama en relación con la pertinencia de la pregunta como factor pedagógico}

En el contexto de la instalación exploratoria de la estrategia didáctica pedagogía jurídica basada en la pregunta implementada por el Programa para la Enseñanza Integral del Derecho y atendiendo el modelo de la investigación-acción-participación, se desarrollaron diversas técnicas con el objetivo de monitorear su desarrollo operativo, además de poder conocer la percepción de los estudiantes en relación con la pertinencia de la metodología utilizada y tanto las capacidades como las limitantes 
existentes para realizar interrogantes durante las clases. Es así como se desarrolla la técnica de la observación participativa, implementándose el diario de campo de los docentes además de encuestas participativas, entrevistas grupales e instrumentos de seguimiento, donde se va sistematizando el avance del proceso de la estrategia didáctica implementada (Rubio, 2016b). Valga decir que son la fuente de las opiniones de los estudiantes las que se dan a conocer en este apartado.

Al sistematizarse la información obtenida de los estudiantes que cursaron la asignatura de Derecho Civil I y II entre 2014 y 2016, mayoritaria y contundentemente reconocen y valorizan que en el ejercicio profesional de un abogado el hecho de realizar e intencionar preguntas es una práctica fundamental, lo cual es necesario comenzar a desarrollar y perfeccionar durante todo su proceso de formación académica (Rubio, 2016b). Al respecto, las percepciones de los estudiantes de derecho se pueden configurar en orden a las siguientes dimensiones.

\section{El abogado como intencionador de preguntas}

En este sentido, los estudiantes tienden a argumentar que el hecho de realizar preguntas le permite a un abogado obtener información y establecer coherencia en su práctica, ya que «a través de una pregunta podría incomodar a la otra parte» (Diario de campo de los docentes, 2014). Además, enfatizan que la pregunta bien direccionada permite al profesional salir de dudas e indican que «también enriquece mucho más su conocimiento [...] algo que los seguirá para toda su carrera, ya que al momento de interrogar a algún testigo o incluso a su propio cliente lo hará con una capacidad casi innata» (Diario de campo de los docentes, 2014).

Las anteriores son algunas de las percepciones referenciadas por los estudiantes que permiten orientar la importancia que le otorgan a instalar una pedagogía cuestionadora en el proceso formativo. De esta forma, añaden que es "profundamente necesario que el abogado haga preguntas cuando está investigando [...] las respuestas a la pregunta que formula un abogado son útiles para resolver problemas en el ámbito judicial, académico, etcétera» (Diario de campo de los docentes, 2014). Por otra parte, señalan que «las preguntas forman parte de una argumentación, ya que hablar con argumentos es importante para la profesión» (Diario de campo de los docentes, 2015).

\section{La desmotivación a preguntar en clases}

A pesar de considerar la relevancia estratégica de ir formándose en una cultura de la pregunta y una lógica cuestionadora, en los tres años analizados aparece un dato ambivalente en la percepción de los estudiantes, ya que una gran mayoría reconoce que ocasionalmente o nunca realizan preguntas en clases (Diario de campo de los 


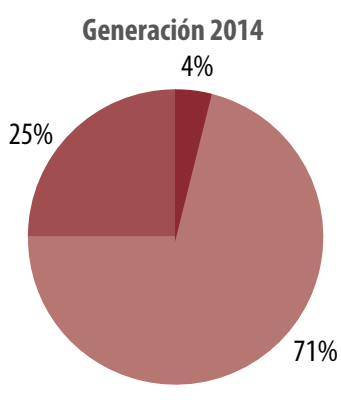

Siempre
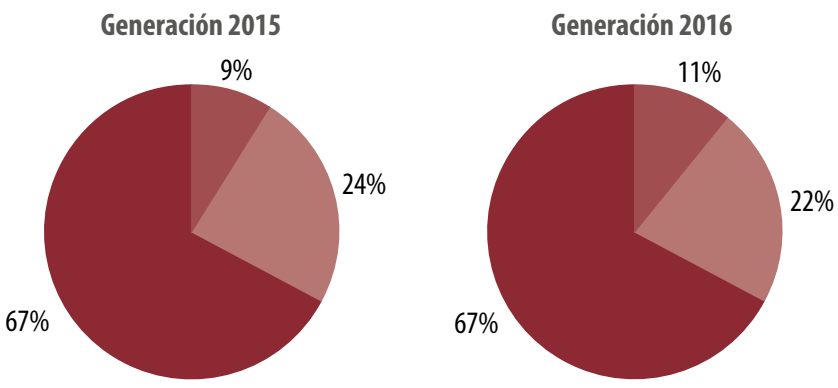

Figura 1. Evolución de la dimensión sobre si se realizan preguntas en el aula.

(Fuente: Rubio, 2016b)

docentes, 2016). Lo anterior se refleja en los resultados de las encuestas realizadas durante el período (ver figura 1), ya que un porcentaje mayoritario de los estudiantes reconoce que «ocasionalmente» 0 «nunca» realizan preguntas en el aula. Sin embargo, la información recabada entrega algunos datos a considerar, ya que el $71 \%$ de la generación del 2014 manifestó que «nunca» realizaba preguntas en clases, porcentaje que disminuyó a un 22\% en la generación del 2016 y que representa un considerable avance al menos en la percepción de participación que manifiestan estas generaciones de estudiantes.

Ante esto, era importante explorar y conocer los principales factores motivacionales que determinan a los estudiantes al momento de efectuar preguntas en clases, es decir, qué los moviliza o no a participar activamente del proceso de enseñanzaaprendizaje. Al respecto, de las encuestas realizadas durante el período emergieron algunos indicadores que en estos tres años lograron cierta estabilidad (ver figura 2). El indicador que motiva a preguntar a este segmento de estudiantes mayoritariamente es el «no entender» la materia planteada en clases, y llama la atención que el indicador de preguntar para «generar debate» fue avanzando de manera progresiva.

En relación con la desmotivación a preguntar, alerta que el indicador «innecesario»y «no hay nada que preguntar» se mantengan estable en estos tres años (Diario de campo de los docentes, 2016). Esto se constata en los resultados de las encuestas realizadas durante tres años consecutivos, donde se le preguntaba a los estudiantes sobre las causas o justificaciones esgrimidas para no realizar preguntas en clases. Para esto se les entregaban los siguientes indicadores: a) por temor; b) por vergüenza; c) por timidez; d) es innecesario; e) porque busco la respuesta solo/a; f) prefiero preguntar a un compañero/a; g) no hay nada que preguntar; h) porque si pregunto el profesor se puede molestar; e i) porque mis compañeros se pueden molestar.

$\mathrm{Al}$ analizar los resultados globales (ver figura 3 ) se puede apreciar que los indicadores que señalan el factor «innecesario» $\mathrm{y}$ «no hay nada que preguntar» representan 


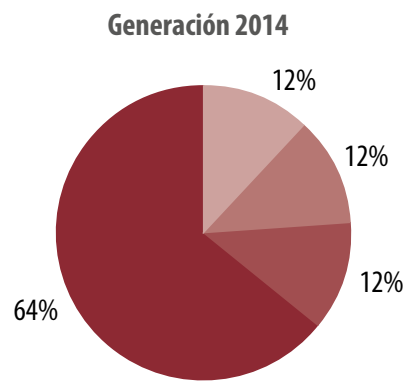

No entiende

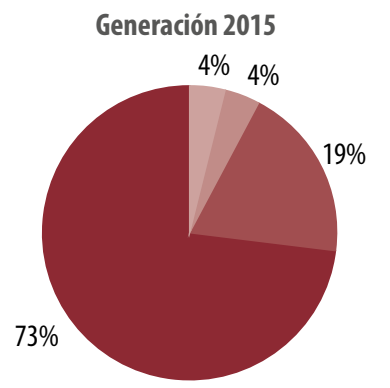

Generar debates

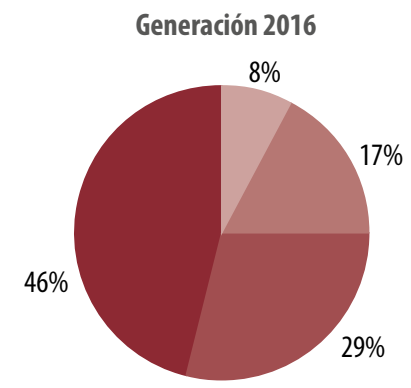

Otros

Figura 2. Evolución de la dimensión sobre la motivación por realizar preguntas en clases.

(Fuente: Rubio, 2016b)

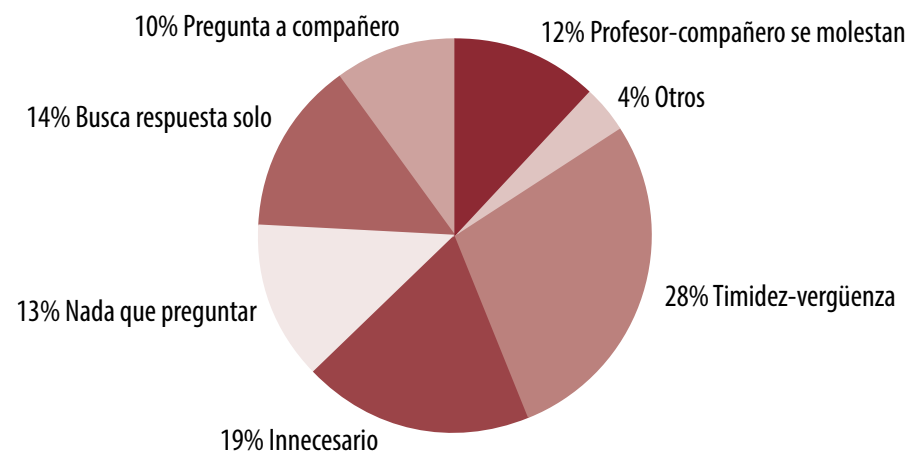

Figura 3. Evaluación de la dimensión sobre la motivación para no realizar preguntas en clases.

(Fuente: Rubio, 2016b)

un $32 \%$ de la percepción de los estudiantes, lo que se visualiza como una paradoja al momento de relacionar estos indicadores con la necesidad que debe manifestar un abogado de «siempre realizar preguntas». Este indicador podría mostrar que el estudiante logra entender mayoritariamente lo explicado en clases, situación que queda en la interrogante, ya que las encuestas arrojan la existencia de factores emocionales y afectivos del estudiante que también estarían en la base de la desmotivación a preguntar (Diario de campo de los docentes, 2015).

En efecto, indicadores como la «vergüenza y timidez» sumado a «buscar las respuestas solos», "preguntar a un compañero» o el «profesor o el compañero se pueden molestar», representan el 64\% de las respuestas de los estudiantes, situación que deja abierta la pregunta de cómo se están abordando las competencias y habilidades afectivas de los estudiantes, base fundamental para propiciar cualquier proceso de enseñanza-aprendizaje entendiendo que la emoción y la cognición son procesos indisolublemente relacionados con el aprendizaje, por cuanto los datos obtenidos esta- 
blecen un campo de acción a considerar en la planificación del proceso educativo a futuro. La paradoja de que por un lado se entienda que un abogado es un intencionador de preguntas y por el otro que no las haga en el proceso de formación, también podría estar explicando porque el estudiante asimila lo expuesto en clases sin realizar revisión epistémica.

\section{Una mediación metodológica que movilice al estudiante cuestionador}

La percepción de los estudiantes señala que en la carrera no se enseña una disposición a la pregunta. Así, este tipo de expresiones son recurrentes en los estudiantes: «nosotros debemos aprender a cómo preguntar por nuestra propia cuenta y no se incentiva» (Diario de campo de los docentes, 2014). Sin embargo, también mencionan lo siguiente: «nos dicen que debemos preguntar, pero nadie educa en el cómo debemos preguntar» (Diario de campo de los docentes, 2014). Sin duda, este es un antecedente importante a considerar, ya que existen percepciones que van más allá: «en la universidad no se enseña a preguntar, ya que dentro de la malla no hay ningún taller o curso especial que nos enseñe a preguntar» (Grupo Focal, 2014).

Dentro de la valorización psicopedagógica de espacios y didácticas surgen dos tipos de percepciones estudiantiles. En primer lugar, están quienes valorizan el proceso, pero con reparos, ya que reconocen que los profesores permiten la acción, pero no median el proceso: «nos da el espacio para realizar preguntas, pero no existe un espacio que nos ayude a desarrollar esta habilidad» (Diario de campo de los docentes, 2014). En segundo lugar, están quienes la critican como inexistente, desacreditando los métodos de enseñanza: «a medida que avanza la carrera, los profesores se centran más en enseñarnos y en que nosotros aprendamos, pero olvidando el cuestionamiento» (Diario de campo de los docentes, 2016). Con esto, se centra la crítica en lo directivo y autoritario del proceso y del docente: «se enojan cuando alguien quiere preguntar algo» (Diario de campo de los docentes, 2015). Por otra parte, también se centra en las didácticas utilizadas: «se vuelve un monólogo o una lectura por parte del profesor, ya sea de un power point o de un texto, no promociona hacer preguntas» (Grupo Focal, 2016).

De acuerdo con las conclusiones generadas por los mismos estudiantes en cuanto a las razones para restringir la pregunta, estos establecen que:

Es necesario preguntar cuando es debido. A veces no se pregunta para no interrumpir la clase. Se debe tener noción del tema que se va a preguntar, es importante autocuestionarse. Se enseña contenido, pero no se enseña a preguntar. Es necesario saber elaborar una buena pregunta y esta se da cuando hay curiosidad, la cual debe saber llevar a una solución (Grupo Focal, 2014). 


\section{Discusión}

Los antecedentes recopilados durante este período de estudio dan cuenta que la percepción de los estudiantes se orienta hacia la carencia de mediación metodológica del proceso, lo que significa que falta desarrollar una intencionalidad estratégica por parte de los docentes que promueva la problematización y no solo otorgar espacios para realizar preguntas. A esto se suma que uno de los factores esenciales que no moviliza a los estudiantes a preguntar se relaciona con la estructura afectiva o alfabetización emocional del aprendiz. Aspectos vinculados a la autoestima y la asertividad sumados a elementos como la timidez, el solucionar sus interrogantes solos o consultar a un compañero aparecen altamente ponderados, a lo que se suman factores relacionados con la autonomía en la línea de que no se les permite argumentar con recursos propios (Rubio, 2016b).

Ahora bien, tras lo revisado se aprecia que existe un convencimiento de que una pedagogía de la respuesta no solo limita la didáctica de la clase, sino que trae implicancias epistemológicas y políticas. Por el contrario, se reconoce que existe en la práctica pedagógica orientada a la pregunta una forma que permite fortalecer la cognición del estudiante hacia áreas más críticas, reflexivas y argumentativas. Entonces, cabe preguntarse por qué no se intenciona el hábito por la pregunta en las aulas de derecho y por qué no se construyen aulas problematizadoras.

Frente a estas interrogantes y los antecedentes que admiten un cuestionamiento directo a las aulas de docencia jurídica tradicionales por parte de la literatura y de los propios estudiantes, como se aprecia en las percepciones revisadas se puede afirmar que: a) se distingue la diferencia entre un docente-facilitador que deja el espacio para preguntas y un docente-tutor que intenciona estratégicamente esta práctica, ante lo cual esto último es más valorado por los estudiantes; b) se afirma que prevalece la transmisión de conocimiento de manera mecánica y directiva sin generar oportunidad para la formación de un estudiante crítico; y c) entre los aspectos relevantes encontrados se puede señalar que para que exista una pedagogía de la pregunta en un aula jurídica deben entenderse integradamente tres fundamentos básicos: los epistémicos, los políticos y los pedagógico-afectivos.

En ese contexto, al analizar el fundamento epistemológico, en relación con el balance del desarrollo de la experiencia de estrategia pedagógica formulada, sumado a la percepción de los estudiantes sobre la misma, y al expresar éstos, al indicador «innecesario» como motivo importante para no preguntar, deja abierta una línea de investigación sobre la relación de ámbitos tales como docente-transmisor y objeto de conocimiento.

En este caso, el qué conocer, con la particularidad del Derecho como contenido, al ser enseñado como un algo acabado, estanco y dogmático, que debe ser memorizado, no permite una apertura dialogante y construible, más bien, representa una verdad 
externa al sujeto que conoce, quien sólo debe asimilarla. Por ello, las dudas o las diferencias de opinión, como que el estudiar, significa para el estudiante, un acto posterior a la clase, cercano y direccionado a la evaluación sumativa resulta un proceso inacabado. Este fenómeno da para una revisión epistémica crítica.

Analizando los fundamentos políticos, si bien las teorías sobre la pedagogía de la pregunta le otorgan a los estudiantes un rol cuestionador, crítico y dialogante, las percepciones de estos - al menos en la experiencia revisada - la reducen a otorgar a la pregunta una connotación de habilidad o competencia, lo que en última instancia la simplifica, ya que puede quedar a nivel de una destreza donde el estudiante puede mantener un rol de sometimiento y producir una castración de la curiosidad (Freire y Faúndez, 2013).

Es curioso que los estudiantes valoren preguntar «a un compañero», entendiéndolo como una dinámica fuera de aula que vendría a enriquecer el contexto social de aprendizaje. En ese sentido, surge la necesidad de comenzar a admitir como presupuesto básico la existencia de la noción de una realidad abierta, es decir, de un contenido dinámico y construible no solo en la sala de clases. Lo anterior surge como factor promotor de una apertura al pluralismo y, con ello, una contribución a la democratización del aula y una herramienta que combata la cultura de la sumisión y del silencio del estudiante. Esto implica poner en tela de juicio la pretensión democratizadora del aula, entendida solo como interacción alumno-profesor con un estudiante predispuesto a responder memorizando contenidos ante lo cual difícilmente podrá pensar, proyectar y teorizar, lo que se suma a un docente habituado a monólogos temáticos, sin capacidad integradora, social ni disciplinaria, quien difícilmente problematizará, sino que será un mero transmisor de información.

En relación con los fundamentos pedagógico-afectivos surge otro elemento pocas veces considerado en la docencia universitaria en general y en la jurídica en particular. Tiene relación con la estructura emocional del estudiante, sobre todo considerando la proyección futura como profesional. En el caso del abogado, se requiere de una estabilidad emocional que le permita un desenvolvimiento funcional, que pueda contraargumentar, debatir con los demás cuestiones que requieren vencer o al menos dominar temores e inseguridades. Si a lo anterior se suma el alto índice de deserción por la angustia de las evaluaciones orales, se abre la posibilidad de admitir que el refuerzo de lo cognitivo-lógico no es separable de una alfabetización emocional, ya que la mayor parte de las percepciones expresadas denotan una falencia en el ámbito afectivo, de la autonomía, motivación y la autoestima; significaciones que representarían un nivel de anulación de sí mismo.

Desde esa perspectiva, surge el convencimiento de que las instituciones educativas deberían prestar mayor atención a la integralidad del proceso de enseñanzaaprendizaje, y no solo atender a la interacción como simple proceso cognitivo lógico de enseñar y aprender ciencias jurídicas u otro contenido de ciencias. Ante esto, se 
evidencia la necesidad de desarrollar prácticas psicopedagógicas que permitan favorecer el desarrollo emocional del estudiante, ya que de otra manera existirán dificultades importantes para cambios psicoeducativos reales.

Como corolario de lo analizado, es conveniente puntualizar que en la incorporación de una pedagogía basada en la pregunta, de acuerdo con los distintos aspectos detectados en el plano teórico y en la experiencia desarrollada, se advierte que la didáctica de la pregunta no es suficiente para la formación de profesionales complejos, críticos y éticos, ya que se requiere un aula democrática tanto en la perspectiva epistemológica de la relación sujeto-objeto-verdad como en la política en sus distintas manifestaciones de poder, docente-estudiante. Esto se debe a que una pedagogía basada en la pregunta no se debe reducir a mejorar esta última como habilidad, sino a desarrollar la estructura emocional y la fortaleza humana necesaria para una formación integral de un estudiante de derecho.

\section{Conclusión}

$\mathrm{Al}$ incorporar la pedagogía basada en la pregunta, la docencia jurídica se aproxima metodológicamente a desarrollar un pensamiento propio, crítico-reflexivo, argumentativo y propositivo en los estudiantes, además de una cognición indagante, analítica, sintética, creativa y capacitada para formar conexiones. Son elementos que claramente se contraponen a los de un estudiante reproductor de contenido, pasivo y disgregador.

En lo revisado anteriormente no solo se evidencian los aportes de una pedagogía basada en la pregunta, sino que además se valida en la percepción de los estudiantes de derecho el reconocer la importancia que debe cumplir la pregunta en el proceso de formación de los abogados. A pesar de esta convergencia, por parte de los docentes e instituciones aún existen prácticas pedagógicas con una connotación epistemológica, política y didáctica que no promueven una alfabetización emocional, y fundamentos complementarios que obstaculizan el logro de estas expectativas y exigencias.

Desde la experiencia de la estrategia didáctica pedagogía jurídica basada en la pregunta, como primera aproximación se intenta establecer una configuración teórica-exploratoria con respecto a un campo - la docencia jurídica-, en el que es necesaria una innovación psicopedagógica que permita posicionar al estudiante de derecho como el verdadero protagonista del proceso de enseñanza-aprendizaje. En ese sentido, intencionar una estrategia pedagógica de este tipo aparece como base mínima e indispensable para la formación de abogados, ya que para este profesional en particular existe una exigencia mayor porque la habilidad de interrogador está -o debería estar-implícita en su práctica natural. Así, se espera que tenga dominio: a) de las normas, porque debe crear argumentos jurídicos entramando normas y no solo realizar un mero trasvasije de datos; y b) debe interrogar la prueba, los hechos, 
testigos, documentos y como creador convencerse a sí mismo y persuadir a los demás de que está a la altura de las expectativas de su cliente y de la aspiración de lo justo. En este sentido, se le exige un hábito por la pregunta como resultado de un aula problematizadora.

Finalmente, es importante señalar que una pedagogía basada en la pregunta para la formación de abogados no solo debe ser concebida como un arte de preguntar como una habilidad y estrategia didáctica, sino también como una actividad transformadora consustancial a un aula problematizadora sustentada en: a) bases epistemológicas que permitan a los sujetos dialogar y construir conocimiento de manera real preparándolos para las incertidumbres y las realidades emergentes; b) bases políticas como parte de la formación de relaciones sociales educativas plurales, lo que se traduce en una formación basada en la ética y, en definitiva, como manera de disponerse afectiva y motivadamente; y c) bases pedagógicas-afectivas, lo que dignifica no solo al estudiante como ser humano, sino a todos los que intervienen en el aula.

\section{Referencias}

Álvarez, Yolanda (2013). «La pedagogía socrática como fundamento de la investigación formativa». Revista Universidad de La Salle, 60: 241-264. Disponible en https://bit.ly/2PKWFEM.

Beneitone, Pablo, César Esquetini, Julia González, Maida Marty, Gabriela Siufi y Robert Wagenaar (2007). Reflexiones y perspectivas de la educación superior en América Latina. Informe Final-Proyecto Tuning América Latina desde 2004 a 2007. Bilbao/Groningen: Universidad de Deusto/Universidad de Groningen.

Betancourth, Sonia, Katherine Insuasti y Nadja Riascos (2012). «Pensamiento crítico a través de la discusión socrática en estudiantes universitarios». Revista Virtual Universidad Católica del Norte, 35: 147-167. Disponible en https://bit.ly/38KHuEt.

Bloom, Benjamin (1976). Características humanas y aprendizaje escolar. Bogotá: Voluntad.

CAPRA, Fritjof (1998). La trama de la vida: una nueva perspectiva de los sistemas vivos. Barcelona: Anagrama.

ELDER, Linda y Richard Paul (2002). El arte de formular preguntas esenciales. California: The Fundation for Critical Thinking.

ELLIOT, Jhon (1994). La investigación-acción en educación. Madrid: Morata.

FACH Gómez, Katia. (2012). «Ventajas del "Problem Based Learning" (PBL) como método de aprendizaje del derecho internacional: Aprendizaje basado en el problema de la inoculación masiva de sífilis en Guatemala». Bordón. Revista de pedagogía, 64 (1): 59-74. Disponible en https://bit.ly/2sBPd6Z.

FreIRE, Paulo y Antonio Faundez (2013). Por una pedagogía de la pregunta: crítica a una educación basada en respuestas a preguntas inexistentes. Buenos Aires: Siglo XXI. 
García, Fernando y Lucía García (2005). La problematización. Etapa determinante de una investigación. México: Instituto Superior de Ciencias de la Educación.

Gómez, Taeli (2014). «Paradigmas emergentes y crisis en la educación del derecho: algo más que didácticas». Revista Pedagogía Universitaria y Didáctica del Derecho, 1 (1): 58-71. DOI: 10.5354/0719-5885.2015.35963

-. (2015). «Andamiar hacia las incertidumbres: Una propuesta educativa-jurídica». Primer Congreso Internacional sobre Investigación en Pedagogía y didáctica del Derecho, Instituto de Investigaciones Jurídicas. Universidad Nacional Autónoma de México.

-. (2018). La complejidad: un paradigma para la educación. Su aporte con una mirada histórica y reflexiva. Santiago: RIL.

GonZÁLEZ, Jorge (2012). «El constructivismo pedagógico aplicado al derecho: hacia una formación dinámica». Boletín mexicano de derecho comparado, 45 (133): 119139. Disponible en https://bit.ly/2Enw4IH.

GonZÁleZ-ReY, Fernando (2000). «El lugar de las emociones en la constitución social de lo psíquico: El aporte de Vigotsky». Educação \& Sociedade, 21 (71): 132-148. Disponible en https://bit.ly/2swZzFl.

ILleRA LobO, Mercedes (2017) «Relación de la teoría y la práctica en la enseñanza del derecho». Revista Espacios, 38 (45): 1-5. Disponible en https://bit.ly/2YSvTid.

LATORRE, Antonio (2003). La investigación-acción. Conocer y cambiar la práctica educativa. Barcelona: Graó.

LóPEZ, Gabriela (2012). «Pensamiento crítico en el aula». Revista Docencia e Investigación, 22: 41-6o. Disponible en https://bit.ly/2PQRBPh.

MARTíneZ, Jorge (2006). «El método socrático en la educación superior». Revista Universidad de La Salle, 42: 86-91. Disponible en https://bit.ly/2PsWGhL.

Mareto Silva, Tatiana y Elda Coelho de Azevedo Bussinguer (2016). «O positivismo como obstáculo epistemológico à produção do conhecimento jurídico: o dogmatismo e suas consequências para a formação do jurista». Revista de Pesquisa e Educação Jurídica, 2 (1): 214-234. Disponible en https://bit.ly/2YVZbMY.

Mastache, Anahí y Roald Devetac (2017). «La formación jurídica: una mirada desde una didáctica analítica y reflexiva». Academia: revista sobre enseñanza del derecho de Buenos Aires, 15 (30): 153-180. Disponible en https://bit.ly/2tkilQE.

Morin, Edgar (2001). El método 1. La naturaleza de la naturaleza. Madrid: Cátedra.

Najmanovich, Denise (2005). «Estética del pensamiento complejo». Andamios, 1 (2): 19-42. Disponible en https://bit.ly/2Ep4fzL.

Osorio, Aura Ximena (2013). «Hacia la excelencia en la educación jurídica». Vniversitas 126: 11-14. Disponible en https://bit.ly/2RWWvwY.

Pupo, Rigoberto (2004a). «La verdad como eterno problema filosófico». Disponible https://bit.ly/3509awN. 
-. (2004b). Imagen, metáfora, verdad. Antología del pensamiento complejo. Tabasco: Universidad Popular de la Chontalpa.

Rubio, Juan (2016a). «Emociones y actividad objetivada, como motor de la construcción del psiquismo humano: una mirada desde el enfoque historicoculturalista». Disponible en https://bit.ly/2thXKwi .

-. (2016b). «Caracterización Sociocultural de la Comunidad Educativa, Carrera de Derecho, UDA». Disponible en https://bit.ly/2r2kodo.

Rivera Ayala, Luis Alonso (2014). «Epistemological relation between Pedagogy, Didactics and Law». Revista de Educación y Derecho, 9: 1-18. Disponible en https:// bit.ly/2trKwxn.

SÁnchez-Puentes, Ricardo (1993). «Didáctica de la problematización en el campo científico de la educación». Perfiles Educativos, (61): 64-78. Disponible en https:// bit.ly/2tkgCLa.

SANTOS, Jackson y Fernanda Macedo (2018). «Reflexões sobre o educador jurista e a concretude da educação jurídica no ensino superior ». Revista de Pesquisa e Educação Jurídica, 4 (1): 95-111. Disponible en https://bit.ly/38LJL2j.

Savater, Fernando (1999). Las preguntas de la vida. México: Ariel.

Schulman, Lee (1986). «Paradigms and Research Programs in the Study of Teaching. A contemporary Perspective». En Merlin C. Wittrock (editor), Handbook of Research on Teaching (pp. 3-36). Nueva York: Macmillan.

TAPIA, Grover (2014). «El contrapunto entre una pedagogía de la respuesta y una pedagogía de la pregunta». Disponible en https://bit.ly/2EqGS8V .

VARGAS, Germán y Emilio Guachetá (2012). «La pregunta como dispositivo pedagógico». Itinerario Educativo, 60 (26): 173-191. Disponible en https://bit.ly/2PWfJ3o.

Vigotsky, Lev (2004). Teoría de las emociones. Estudio histórico-psicológico. Madrid: Akal.

Villasante, Tomás, Manuel Montañés y Joel Martí (200o). La investigación social participativa. Construyendo ciudadanía. Barcelona: El viejo topo.

Zuleta, Orlando (2005). «La pedagogía de la pregunta. Una contribución para el aprendizaje». Educere, 9 (28): 115-119. Disponible en https://bit.ly/2PUC5Sq.

\section{Sobre los autores}

Taeli Gómez Francisco es abogada, doctora en Ciencias Filosóficas por La Universidad de La Habana en Cuba, creadora y directora del Programa para la Enseñanza Integral del Derecho (PEID) del Departamento de Ciencias Jurídicas de la de la Universidad de Atacama en Chile, donde es académica titular y dicta la cátedra de Filosofía del Derecho y Ética. Su correo electrónico es taeli.gomez@uda.cl. (D) http:// orcid.org/0000-0001-8081-1417. 
Juan Rubio González es psicólogo, licenciado en Psicología, diplomado en Psicología Educativa y en Pensamiento Complejo en la Multiversidad Mundo Real Edgar Morin de México. Actualmente se encuentra maestrando en Investigación y Docencia en Psicología en la Universidad de Atacama en Chile. Además, es investigador, docente y profesional de apoyo metodológico del (PEID). Su correo electrónico es juan.rubio@uda.cl. (D) https://orcid.org/oooo-0001-8118-5104.

Walter GonzÁLEZ Morales es abogado y actualmente se encuentra maestrando en Educación. Además, es diplomado en Docencia e Investigación Universitaria y académico de la Facultad de Ciencias Jurídicas y Sociales de la Universidad de Atacama en Chile. Su correo electrónico es walter.gonzalez@uda.cl. (D) https://orcid. org/0000-0002-5907-8473. 
La Revista Pedagogía Universitaria y Didáctica del Derecho (RPUDD) es una publicación científica semestral que contribuye a la reflexión multidisciplinaria sobre pedagogía universitaria y didáctica del derecho, para la formación y consolidación de esta área de investigación; así como a la difusión de prácticas innovadoras en la enseñanza-aprendizaje del derecho considerando el contexto nacional e internacional. Es una publicación electrónica internacional con una codirección entre Brasil y Chile.

\author{
DIRECTORA \\ María Francisca Elgueta Rosas \\ Universidad de Chile \\ DIRECTOR \\ Renato Duro Dias \\ Universidad Federal de Rio Grande, Brasil \\ SITIO WEB \\ pedagogiaderecho.uchile.cl \\ CORREO ELECTRÓNICO \\ rpedagogia@derecho.uchile.cl \\ LICENCIA DE ESTE ARTÍ́CULO
}

Creative Commons Atribución Compartir Igual 4.0 Internacional

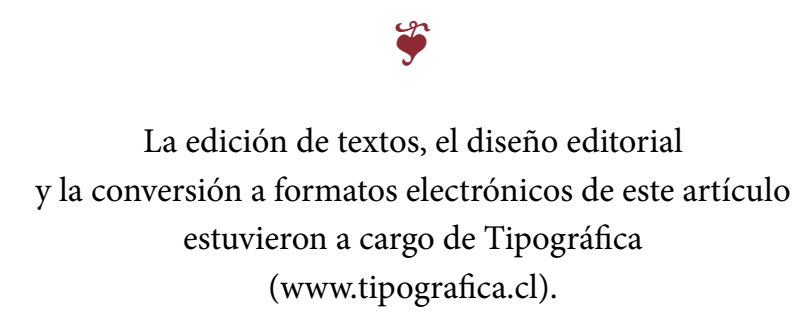

